\title{
Congenital coronary artery anomalies in adult population detected using dual source ECG-gated CTA in a single institution
}

\author{
R.T. Mathai ${ }^{1}$, D.M. Fahmy ${ }^{1}{ }^{2}$, H.L. Sadek ${ }^{3,4}$, W.M. Renno 4 \\ ${ }^{1}$ Department of Radiology Department, Dar Al-Shifa Hospital, Kuwait \\ ${ }^{2}$ Department of Diagnostic Radiology, Faculty of Medicine, Mansoura University, Egypt \\ ${ }^{3}$ Department of Anatomy, Faculty of Medicine, Sohag University, Egypt \\ ${ }^{4}$ Department of Anatomy, Faculty of Medicine, Kuwait University, Kuwait
}

[Received: 29 May 2016; Accepted: 6 July 2016]

Background: Congenital anomalies of the coronary arteries (CAs) are rare and are often diagnosed incidentally during a conventional coronary angiography. Recently, the incidence of these congenital defects is on the rise particularly after the introduction of the electrocardiography (ECG) gated coronary computed tomographic angiography (CCTA). This innovative radiological screening modality has led to the most precise mapping of the course of the CAs on computed tomographic scan. The aim of the study is to determine the prevalence and describe the CAs congenital anomalies and their variations in Kuwaiti population at a single institution experience. Materials and methods: We analysed the CCTA data obtained consecutively from 842 patients (2013-2014), retrospectively. The inclusion criteria for patients' selection were: atypical chest pain, equivocal ECG, assessment of patency of coronary stents or grafts and pre-operative screening. Information was acquiesced using a dual-source CT scanner with ECG gating.

Results: Data analysis revealed that $22(2.61 \%)$ patients were found to have CA anomalies out of the 842 patients who underwent CCTA. Out of these CA anomalies, 13 cases showed more than two ostia, 7 cases showed the ectopic origin of a CA from opposite sinus or non-aortic sinus, 2 cases showed single coronary ostium and 1 case showed coronary artery with pulmonary fistula. Also, myocardial bridging was identified in 78 (9.26\%) patients whereas ramus intermedius branch was identified in 160 (19\%) patients.

Conclusions: The prevalence of CA anomalies in Kuwait was 2.6\%, which is relatively higher than previously reported studies from different countries. (Folia Morphol 2017; 67, 2: 208-218)

Key words: congenital anomalies, coronary arteries, CCTA, prevalence, computed tomography scan

\section{INTRODUCTION}

Congenital anomalies of the coronary arteries (CAs) are uncommon with a prevalence of $1 \%$, but figures between $0.3 \%$ and $5.6 \%$ were reported in the literature $[4,5,7,35]$. The diagnosis of coronary artery anomalies (CAAs) is usually established by invasive coronary angiography (ICA). However, due to the two-dimensional (2D) projection nature of ICA, the visualisation of a complex three-dimensional (3D) vessel course as well as clarification of the exact re-

Address for correspondence: Prof. W.M. Renno, Department of Anatomy, Faculty of Medicine, Kuwait University, P.O. BOX 24923, Safat 13110 Kuwait, tel: 009652463 6283, fax: 00965 25319478, e-mail: wrenno@hsc.edu.kw 
lationship to surrounding anatomical structures may be difficult, and misinterpretation is reported in up to $50 \%$ of the cases $[20,35]$. Recently, coronary computed tomography angiography (CCTA) has emerged as a noninvasive alternative for the evaluation of the CAs [14], particularly after the introduction of modern protocols $[1,18]$ that allowed performing CCTA with a radiation dose substantially lower than that of ICA [19]. Therefore, CCTA has been recommended as the first-line method for the assessment of known or suspected coronary disease [33].

Most of the CAAs have been detected incidentally during the evaluation of patients with suspected CA disease. Although CAAs lack clinical significance in the majority of these patients, certain abnormal patterns, like the anomalous origin of a coronary vessel from the opposite sinus have been associated with sudden cardiac death and ischaemic complications $[4,7,20]$. In $17 \%$ of athletes' deaths, $12 \%$ of sport-related deaths in 14- to 40-year-old individuals are due to CAA $[4,5]$.

The aim of the work was to determine the prevalence and describe the CAA in referred patients undergoing CCTA in Kuwaiti community in a single institution.

\section{MATERIALS AND METHODS}

Eight hundred forty-two (842) patients were referred to the Department of Radiology between April 2013 and September 2014 at Dar-Al-Shifa Hospital, Kuwait City, Kuwait for cardiac multi-detector CT angiography (MDCTA). The main complaints were atypical chest pain, equivocal electrocardiogram (ECG) in addition to patients referred for assessment of patency of coronary stents or grafts and pre-operative screening. All the 842 patients' data sets were reviewed in search of CAA in the origin and course of the vessels.

\section{Demographic analysis}

Out of 842 patients, 446 (52.97\%) were male, and $396(47.03 \%)$ were female. The age range of the subjects was between 24 and 88 years with a mean age of $44.0 \pm 12.3$ years.

The institutional ethical committee approved data analysis at Dar Alshefaa Hospital.

\section{MDCTA technique}

Dual-source CT-coronary angiography was performed using a Siemens SOMATOM Definition Flash dual-source scanner (Siemens Medical Solutions, Forchheim, Germany). With two tubes and two detectors mounted at orthogonal orientation in the gantry, temporal resolution significantly improved. A gantry rotation time of $0.28 \mathrm{~s}$ thus results in a temporal resolution of $75 \mathrm{~ms}$. Tube voltage for CT-angiography was 100-120 kV for both tubes, current $2110 \mathrm{~mA}$ with modulation, the full current between $30 \%$ and $80 \%$ of the cardiac cycle, pitch $0.2-0.44$ adapted to the high resolution. Per-rotation 128 slices are generated with a collimation of $0.6 \mathrm{~mm}$, leading to an isotropic voxel resolution of approximately $0.6 \mathrm{~mm}$ edge length and $0.2 \mathrm{~mm}^{3}$ volume. Radiation dose was adjusted not to exceed $350 \mathrm{mGy} \mathrm{cm}$.

No preparation or beta-blockers were given before the examination. Non-contrast scan for calculation for coronary calcium score was taken from the carina to the apex of the heart. The scan was followed by a test bolus injection to calculate the peak of contrast enhancement time. Then the final coronary angiogram was taken.

A bodyweight adapted volume of contrast agent $\left(1.25 \mathrm{~cm}^{3} / \mathrm{kg}\right.$ body weight; Xenetix 350, Guerbet, Nederland) was injected continuously at a calculated rate $(6.5 \mathrm{~mL} / \mathrm{s})$ to achieve constant injection duration of the $20 \mathrm{~s}$. A saline flush $\left(100 \mathrm{~cm}^{3}\right.$ at $\left.5 \mathrm{~cm}^{3} / \mathrm{s}\right)$ was applied to maintain a compact bolus.

Axial images were reconstructed with $0.75 \mathrm{~mm}$ slice thickness and $0.5 \mathrm{~mm}$ increment using a medium sharp convolution kernel (B26) and retrospective ECG gating. The reconstructions were performed in $5 \%$ steps over the entire R-R cycle using a single-segment algorithm that utilises a quarter segments of projection data from both detectors.

\section{Image analysis}

All acquired MDCTA images were transferred to a dedicated CT 3D-post-processing workstation (Syngo via, Siemens Healthcare Global, Malvern, PA). Two experienced radiologists evaluated the CAs by performing the maximum intensity projections (MIP), curved multiplanar reformats, and volume rendering technique. The CAA were classified into anomalies of origin (number of coronary ostia, high take-off, ectopic coronary origin), anomalies of course (myocardial bridging) and anomalies of termination (CA fistula).

\section{RESULTS}

\section{Image analysis}

The different abnormalities were categorised into four different anomalies: anomalies of origin, anomalies of course, anomalies of termination, and CA variants. Table 1 is a summary of the number and percentage of subjects in each category. 
Table 1. Categories and percentages of the different abnormalities reported in this study

\begin{tabular}{llc}
\hline $\begin{array}{l}\text { Categories of different } \\
\text { anomalies }\end{array}$ & Description & $\begin{array}{c}\text { No. of cases } \\
(\%)\end{array}$ \\
\hline Anomalies of origin & Multiple ostia & $13(1.54 \%)$ \\
& Ectopic coronary origin & $7(0.83 \%)$ \\
& Single coronary ostium & $2(0.24 \%)$ \\
& Total & $22(2.61 \%)$ \\
Anomalies of course & Myocardial bridging & $78(9.26 \%)$ \\
Anomalies of termination & $\begin{array}{l}\text { Fistulous tract } \\
\text { Connection branches }\end{array}$ & $1(0.12 \%)$ \\
& $\begin{array}{l}\text { Ramus intermedius } \\
\text { branch }\end{array}$ & $160(19.00 \%)$ \\
\hline
\end{tabular}

\section{Anomalies of origin}

Multiple ostia were noted in 13 (1.54\%) cases. Eight cases showed a separate origin of the conal artery from the right coronary sinus (CS) (Fig. 1A). While 5 cases showed the absent of the left main CA with a separate ostium for both left anterior descending (LAD) [also known as anterior descending branch of left coronary artery or anterior descending branch] and left circumflex artery (LCX) [also known as circumflex artery or circumflex artery of left coronary] from the left CS. Curved multiplane reformat image (Fig. 1B) showed an absent left main CA. Instead, the LAD and LCX originated from a common ostium of the left CS. The volume-rendered image (Fig. 1C) showed an absent left main CA with a separate origin of LAD and LCX from the left CS.
Ectopic coronary origin was noted in 7 (0.83\%) cases. Four of these cases showed right coronary artery (RCA) originating from the left CS with separate ostium, and then it runs inter-arterial course between the root of the aorta (Ao) and pulmonary artery (PA). Figures 2A (volume rendered) and Figure 2B-D (sagittal multiplanar reformat) images showing the RCA originating from the left CS then runs in the inter-arterial course between the root of the Ao and PA.

Our data analysis showed that in 1 case, the left main CA originated from the posterior aortic sinus (non-CS). The left main CA then runs posteriorly in the retro-aortic course between the Ao and left atrium (LA) as shown in the volume-rendered image (Fig. 3A) and the axial source images (Fig. 3B-D). Likewise, only in one case, the LCX originated from the right CS with separate ostium. The LCX then took a retroaortic course between the aorta and LA to reach the atrioventricular (AV) groove as shown in the volume rendering image (Fig. 4A) and sagittal multiplanar reformat images (Fig. 4B-D).

In $2(0.26 \%)$ cases, a single coronary ostium was found. In the first case the left main CA originated from the right CS by common ostium with the RCA, then took a long retro-aortic course to reach the anterior aspect of the heart between the Ao and LA where it divided into $L A D$ and LCX (Fig. 5A, B). The other case showed a single left main $C A$ with no right CA originating from the Ao. The RCA originated from
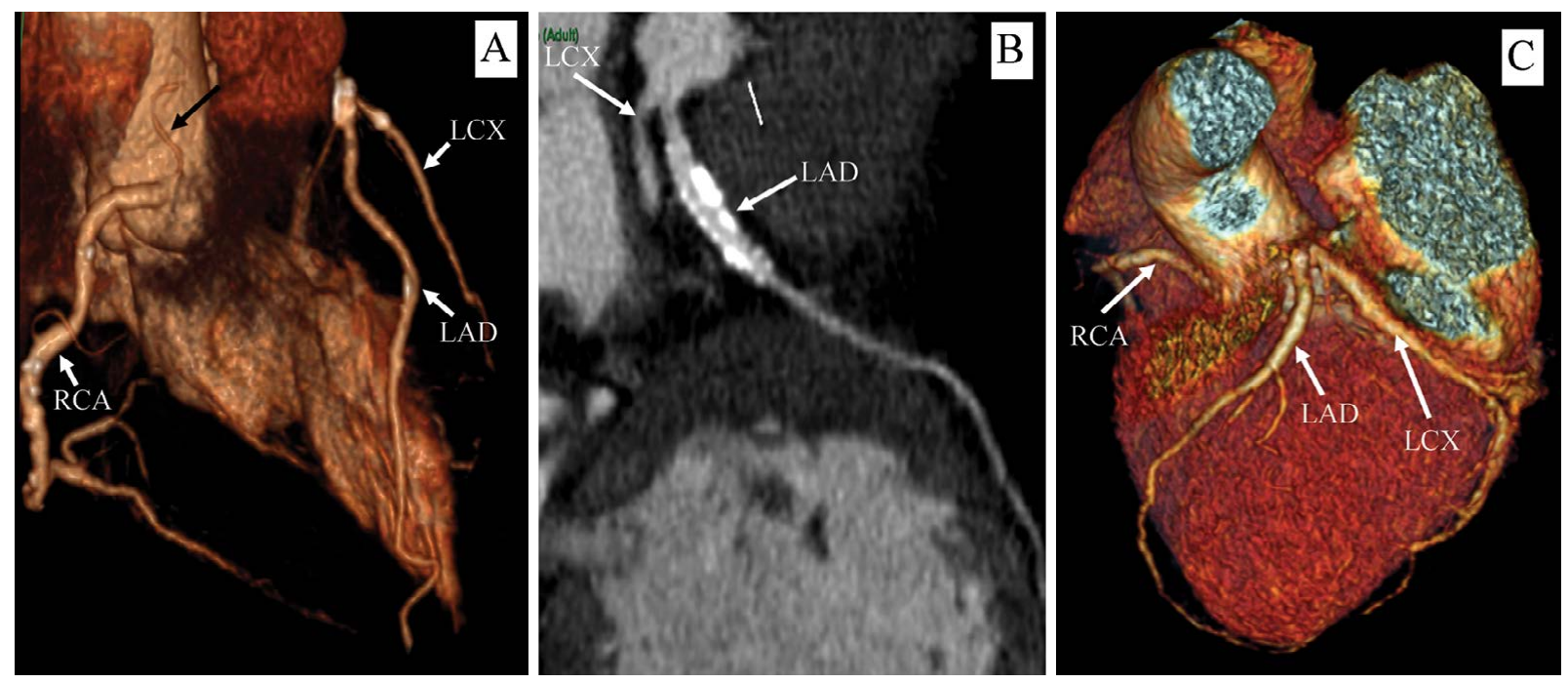

Figure 1. A. Volume rendered image revealed the separate origin of a conal branch from the right coronary ostium (black arrow); B. Curved multiplanar reformat image showing an absent left main coronary artery. Instead, the left anterior descending artery (LAD) (stented) and left circumflex artery (LCX) originated from a common ostium from the left coronary sinus; C. Volume rendered image showing an absent left main coronary artery with the separate origin of $L A D$ and $L C X$ from the left coronary sinus; $R C A$ — right coronary artery. 

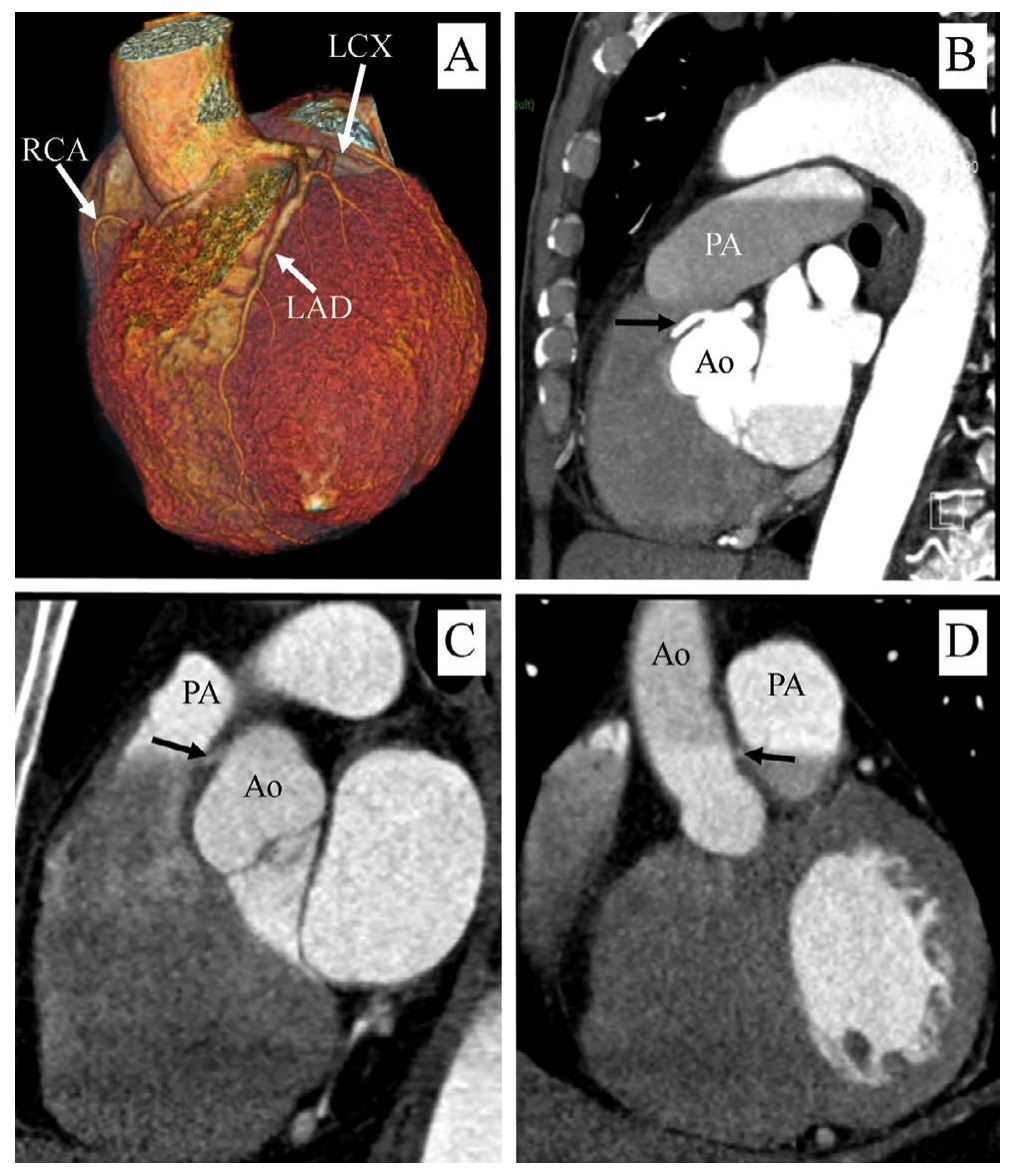

Figure 2. A. Volume rendered; B-D. Sagittal multiplanar reformat images revealed right coronary artery (RCA) (black arrow) is originating from the left coronary sinus then runs in the inter-arterial course between the root of the aorta $\left(\mathrm{A}_{0}\right)$ and pulmonary artery (PA); LAD - left anterior descending artery; LCX - left circumflex artery.
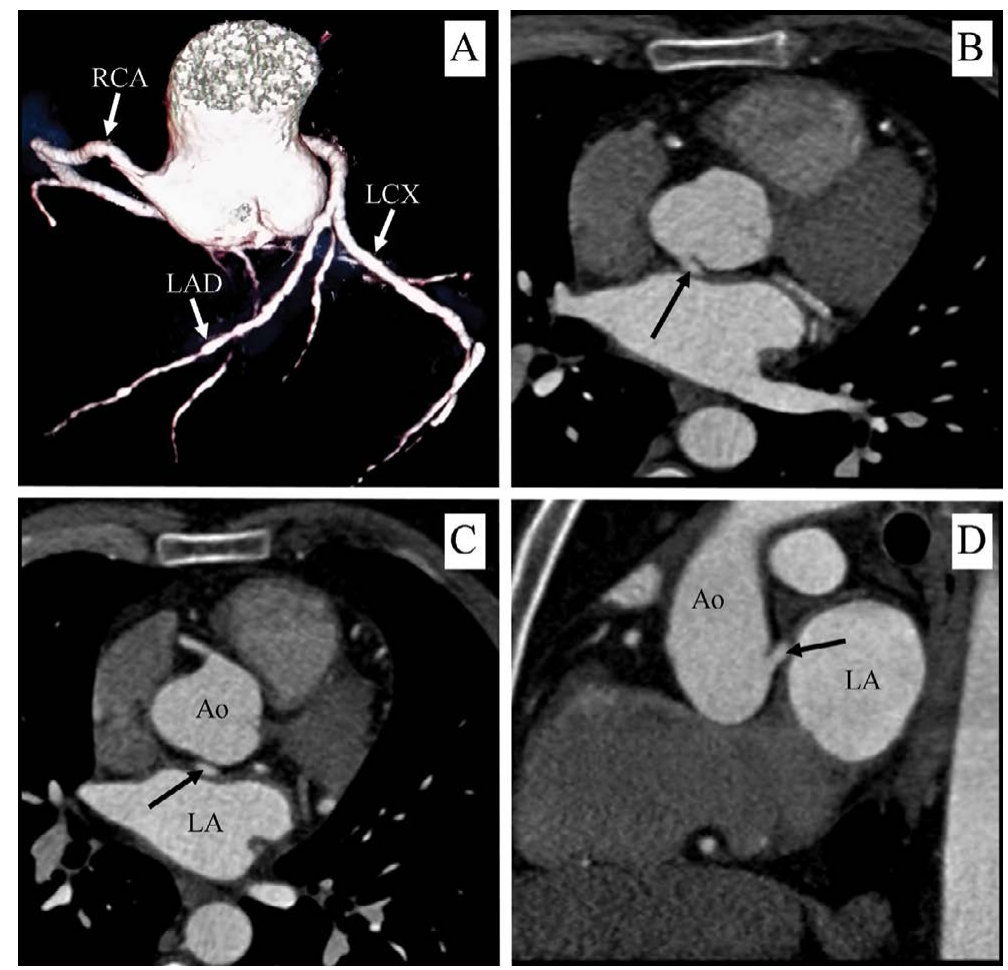

Figure 3. A. Volume rendered; Axial source images (B, C) and sagittal multiplanar reformat images (D) showing that the left main coronary artery (LAD) is arising from a non-coronary sinus (the posterior sinus), then runs in the retro-aortic course (black arrows); Ao - aorta; LA — left atrium; LCX — left circumflex artery; RCA right coronary artery. 

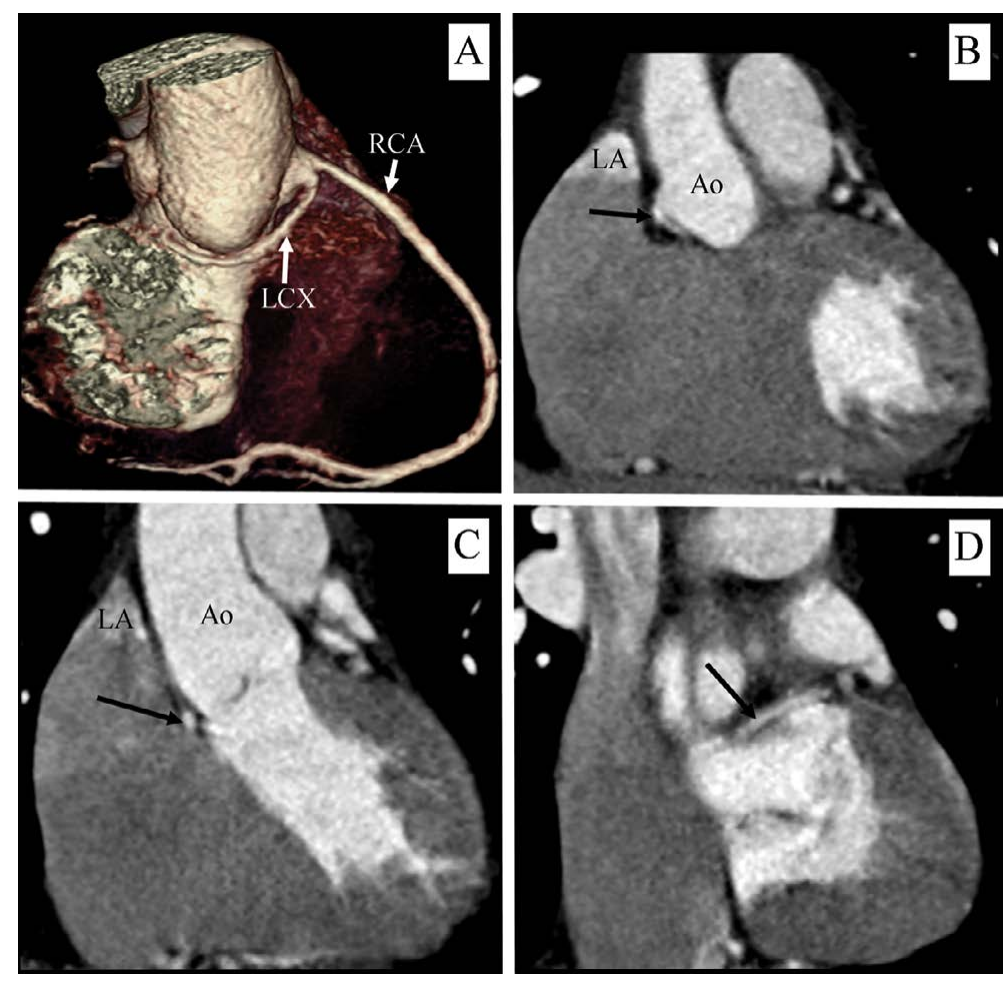

Figure 4. A. Volume rendered; B-D. Sagittal multiplanar reformat images revealed that the left circumflex artery (LCX) had separate origin from the right coronary sinus, then took the retro-aortic course (black arrows) between the aorta $(\mathrm{Ao})$ and left atrium (LA); RCA — right coronary artery.
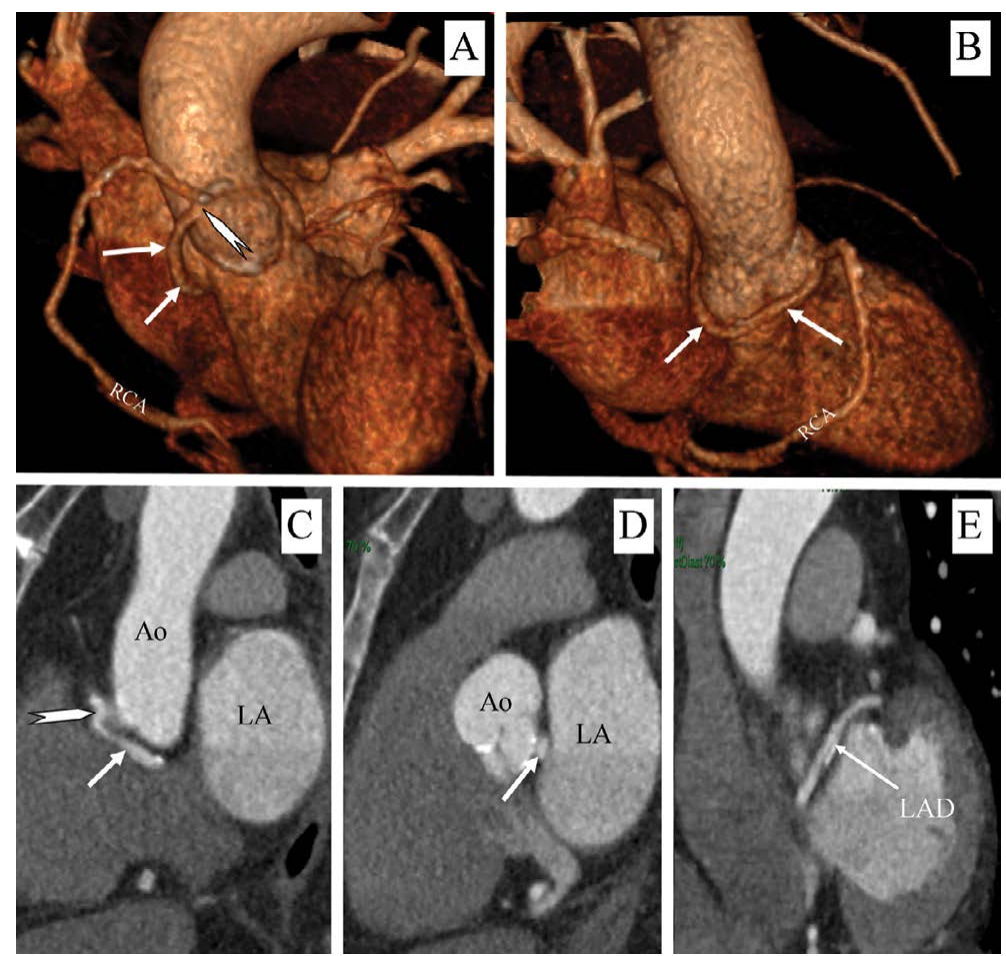

Figure 5. A, B. Volume rendered images; C, D. Sagittal multiplanar reformat images and coronal reformat image revealed that left main coronary artery (white arrows) originated from the right coronary sinus by common ostium with the right coronary artery (RCA) (white chevron) then took a retro-aortic course between the aorta $(\mathrm{Ao})$ and the left atrium (LA); E. Left anterior descending artery (LAD) coursing anteriorly to reach the anterior interventricular groove.

the proximal LAD, and it took an anterolateral course to reach the right lateral margin of the heart in the anterior interventricular groove (Fig. 6A, B).

\section{Anomalies of course}

Our data analysis detected myocardial bridging in $78(9.26 \%)$ cases. All were identified at mid LAD. 

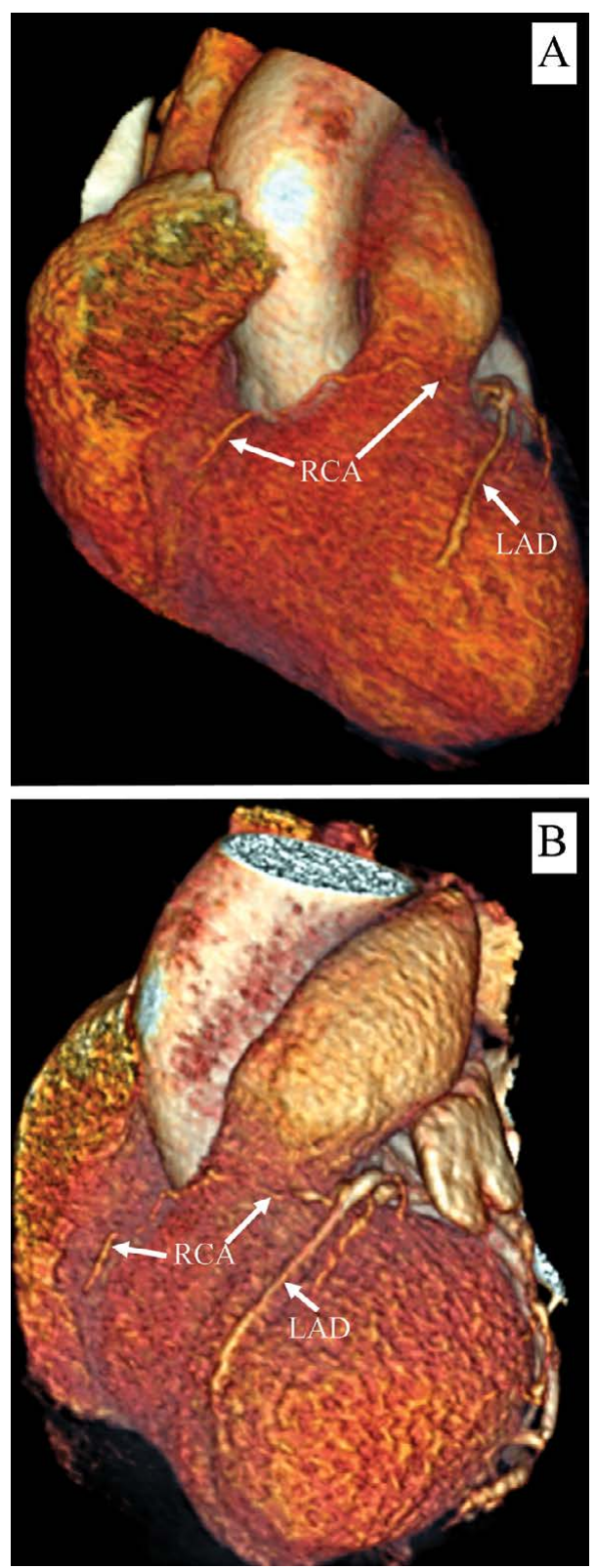

Figure 6. A, B. Volume rendered images revealed a single coronary artery originating from the left coronary sinus. The right coronary artery (RCA) took origin from the proximal left anterior descending artery (LAD).

Figure 7A (MIP image) and Figure 7B (sagittal multiplanar reformat image) taken from different patients showing examples of myocardial bridging of mid-LAD. These cases were further subdivided according to depth of bridging either $<1 \mathrm{~mm}$ (superficial; $\mathrm{n}=53$ ) or $>1 \mathrm{~mm}$ (deep; $\mathrm{n}=25)$.

\section{Anomalies of termination}

Only $1(0.12 \%)$ case had fistulous tract connection branches from the RCA and LAD to small common conduit connected to the PA. The volume rendered
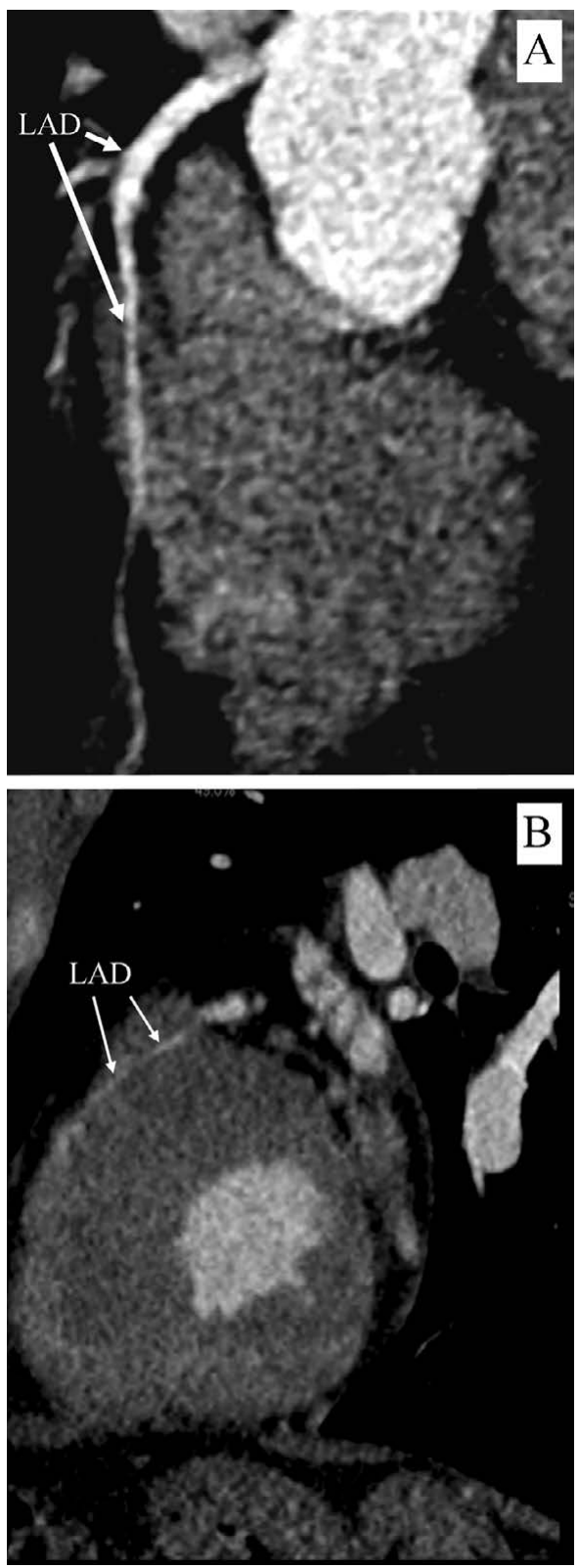

Figure 7. A. Maximum intensity projection image; B. Sagittal multiplanar reformat image (from a different patient) revealed myocardial bridging of mid-left anterior descending artery (LAD; white arrows).

images revealed a coronary fistula supplied by a tortuous septal branch from LAD (Fig. 8A) and conal branch (Fig. 8B). Meanwhile, the sagittal multiplanar reformat image showed that both feeders end in a small vascular channel connected to an anterior aspect of the main PA (Fig. 8C).

\section{Coronary artery variants}

In $160(19.00 \%)$ subjects, a ramus intermedius branch was detected as CA variants. A curved multiplanner reformat image (Fig. 9) shows a trifurca- 

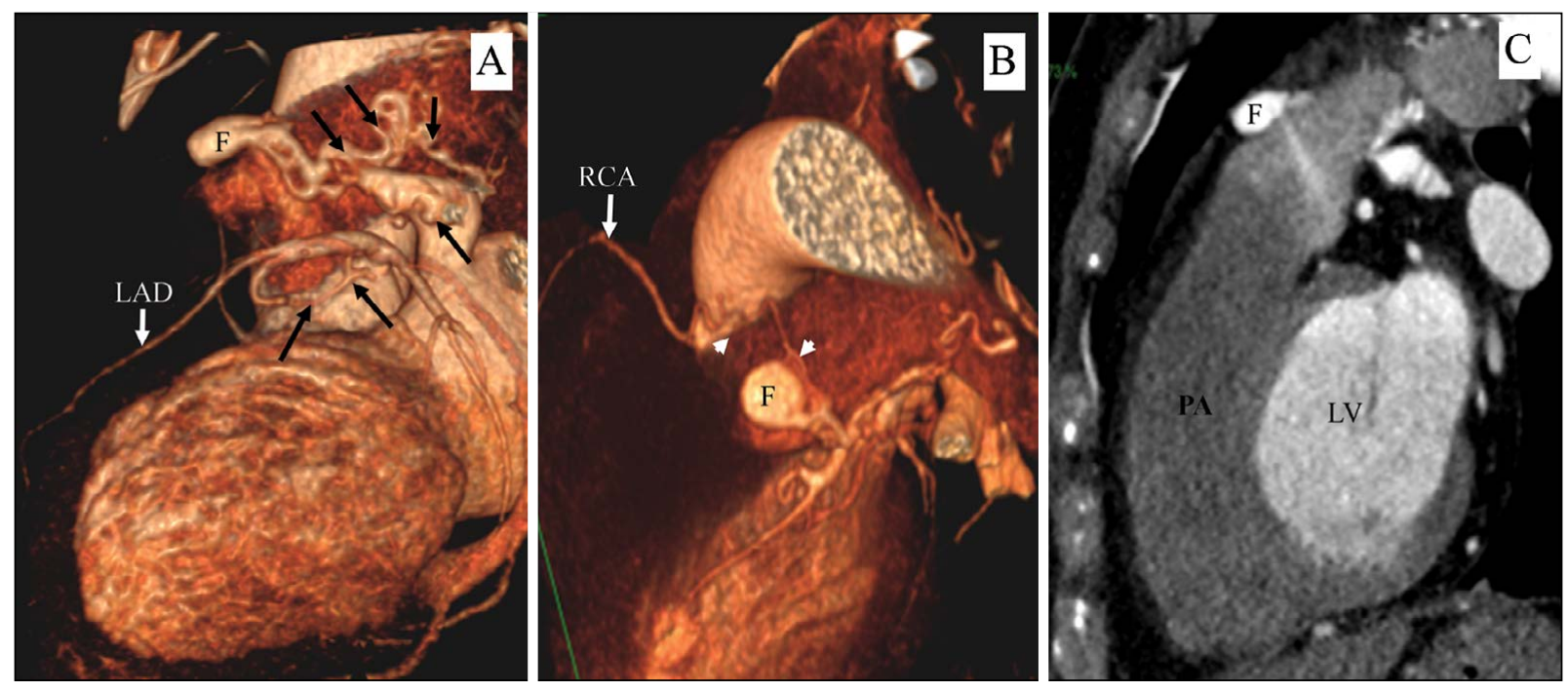

Figure 8. A, B. Volume rendered images and revealed coronary fistula (F) supplied by a tortuous septal branch from LAD (black arrows) and conal branch (white arrowheads); C. Sagittal multiplanar reformat image showing that both feeders end in a small vascular channel (F) connected to an anterior aspect of the main pulmonary artery (PA); LAD — left anterior descending artery; LV — left ventricle.

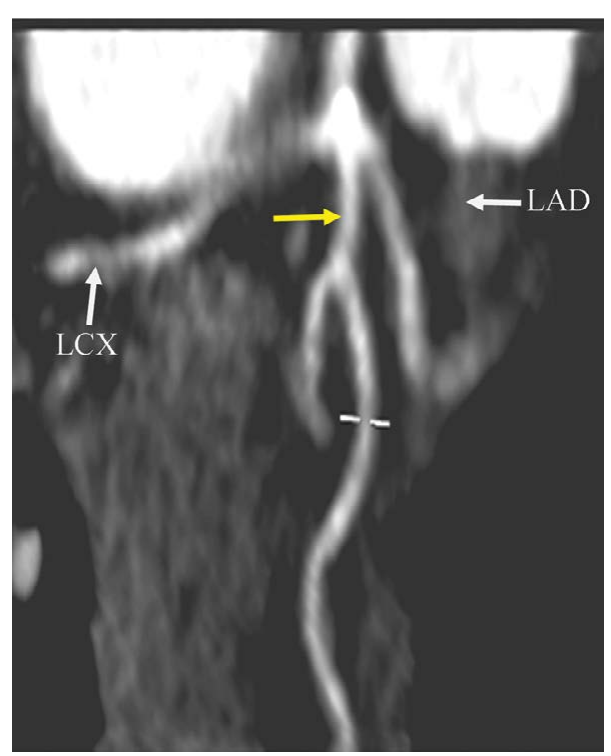

Figure 9. Curved multiplanner reformat image showing a trifurcation of the left main coronary artery into left anterior descending artery (LAD), left circumflex artery (LCX) and ramus intermedius (yellow arrow).

tion of the left main CA into LAD, LCX and ramus intermedius.

Regarding the dominance CAs anatomy (Table 2), $706(83.85 \%)$ cases showed right dominance, 75 (8.91\%) cases showed left dominance and 61 (7.24\%) cases showed co-dominance.

\section{DISCUSSION}

The overall prevalence of congenital CAA in our study is $2.6 \%$. This percentage prevalence includes
Table 2. Percagates of the different coronary arteries dominance

\begin{tabular}{lcc}
\hline Dominance coronary arteries & No. of cases & Percentage \\
\hline Right dominance & 706 & $83.85 \%$ \\
Left dominance & 75 & $8.91 \%$ \\
Co-dominance & 61 & $7.24 \%$ \\
\hline
\end{tabular}

cases of anomalous origin and course as well as cases of anomalous termination. Myocardial bridging was not included in the prevalence calculation as it was excluded from most of the previous studies in the literature. Although our results are near to the results of von Ziegler et al. [38] who reported a prevalence of $2.3 \%$ yet it is still high as compared to other studies $[36,40]$. The high prevalence in this study may be attributed to the greater advances in imaging that made it less invasive and easier to image CAs accurately our patient population. Another reason is that coronary $\mathrm{CT}$ is considered as part of the preoperative assessment in our institution, which allowed us to examine a large number of asymptomatic patients and, in turn, expanded the prospects of detecting congenital anomalies.

Some classification systems of the CAA have been suggested $[4,22,24]$. According to these systems, anomalies of the CAs are divided into anomalies of origin and course, anomalies of intrinsic CA anatomy, and anomalies of termination. In our study, we used the anomalies of origin, course, and termination in addition to the CA variants. Some authors consider the multiple ostia as normal variants [41]. While oth- 
ers include it under the category of anomalous origin $[31,42]$, as we did in the current study. We detected $8(0.95 \%)$ cases of separate origin of the conal branch from the right CS (Fig. 1A) while $5(0.6 \%)$ cases showed absent of left main CA. Instead, the LAD and LCX had separate ostia from the left sinus of Valsalva (Fig. 1B, 1C) which is slightly higher than previous studies $[11,27]$. Multiple ostia may not cause major clinical problems, but they may cause difficulty in cannulating the vessels during coronary angiography [42].

An anomalous CA can arise from the non-CS or the opposite sinus. The abnormal CA then takes one of the four following paths depending on the anatomic relationship of the anomalous vessel to the Ao and the pulmonary trunk. It may proceed into inter-arterial (between the aortic root and the pulmonary trunk) (Figs. 2, 3), retro-aortic (dorsal to theaortic root) (Figs. 4, 5), pre-pulmonic (anterior to the PA or right ventricular outflow tract), trans-septal or sub-pulmonic (beneath the right ventricular outflow track) course [24]. In our sample population, we observed 4 cases of anomalous RCA origin from left CS with inter-arterial course between the aortic root and PA (Fig. 2). Although many patients are asymptomatic at the time of presentation or diagnosis, surgical correction is recommended due to the risk of ischaemic sudden death [9].

We had 1 case in which the left main coronary originated from non-CS with the retro-aortic course (Fig. 3). Moreover, in another patient the LCX originated by a separate ostium from the right CS with a retro-aortic course to reach AV groove (Fig. 4). In a CA surgery study, Click et al. [10] reported that left main coronary originating from non-CS with the retro-aortic course is the most common anomaly of LCX origin as it was detected in $69 \%$ while LCX originated as a branch of the RCA in the remaining cases. LCX origin anomaly could be isolated (as in our case) or associated with anomalous origin of the LAD [15].

The anomalous coronary ostium originating from the unassigned aortic sinus has a slit-like shape that differs from a normal round coronary ostium [37]. The presence of anomalous course between great vessels is, even more, relevant to the increased clinical jeopardy than is the ectopic origin of CA [37, 39]. The increased risk is due to the squeeze of a CA by the increased cardiac output during diastolic expansion of the vessels [7]. Moreover, active exercise may increase the preexisting acute angulations of the CAA [28].
Abnormal RCA origin and course is associated with higher prevalence of cardiac events as compared to anomalous LAD and LCX [12]. Single CA is a situation where the LMCA and RCA arise with a common ostium from the right, left or non-CS. This abnormality is a very rare congenital CAA that is seen in only $0.024-0.044 \%$ of the population [2]. The single anomalous trunk supplies blood to the entire heart and is classified according to its origin, branching pattern, and course. In the current study, we had 1 case with the left main CA originated from the right CS through a common ostium with the RCA. At that point, the LMCA took a long retro-aortic course to reach the anterior aspect of the heart where it divided into the LAD and LCX (Fig. 7). In another case with a single left coronary ostium, the left main CA directly originated from the ostium while the RCA originated from the proximal LAD (Fig. 8).

In this study, the presence of a single CA can be an isolated finding (as in our 2 cases) or associated with additional cardiac anomalies [2]. As previously mentioned with the ectopic origin of the $C A$, the course taken by the vessels after the division of single vessel to right and left has more clinical significance [32].

Myocardial bridging (MB) is a congenital condition in which a segment of major epicardial CA proceeds intramurally through the myocardium beneath the muscle bridge [3]. The MB was the most frequently found anomaly as it was diagnosed in $78(9 \%)$ cases in the current study. In contrast, Shabestari et al. [34] found much higher incidence (21.3\%).

Myocardial bridging is considered a benign condition that most commonly affects the middle segment of the LAD [3] as shown in Figure 9. However, the relationship between $\mathrm{MB}$ anomaly and increased cardiovascular morbidity is still unclear. According to previous reports [21,30], the MB may cause coronary heart disease by two mechanisms. First, by a direct systolic compression of the tunnelled segment and second by the enhanced atherosclerotic plaque formation occurring because of alteration of haemodynamic factors, mainly in the vessel segment proximal to the bridge. The length, the depth and the location of the MB have been associated with the mechanisms above $[21,30]$. Therefore, MB is classified as superficial or deep, depending on the thickness of the covering muscular layer $(\leq 1 \mathrm{~mm}$ or $>1 \mathrm{~mm}$ ) [26]. Also, superficial MB can be classified as complete or 
incomplete by the extent of the vessel encasement by the myocardium [23]. In the current study, among the 78 patients with MB, 25 (32\%) cases were deep bridging, and $53(68 \%)$ were superficial which is similar to the results of Kim et al. [24].

Coronary artery fistulas are present in $0.002 \%$ of the general population and are visualised in nearly $0.25 \%$ of the patients undergoing cardiac catheterisation [16]. The majority of the fistulas drain into the PA or right ventricle, but fistulae may also drain into anywhere from between the vena cava or CS to the PA or LA [6]. Multiple fistulae may also occur. Over $90 \%$ of fistulae drain into the right side of the heart causing right to left shunt. Chronic large volume shunts through these fistulae may cause major aneurysmal enlargement of the proximal feeding CA in addition to enlargement of the receiving vessel or chamber. The uninvolved CAs are usually normal [29]. A study by Zhou et al. [44] reported that fistulae more commonly (50.0\%) originate from both the LCA and the RCA followed by the LCA in $30.4 \%$ and RCA in $12.0 \%$. The remaining $7.6 \%$ of the cases were associated with extra-cardiac communications.

In the current study, we encountered 1 case of coronary-pulmonary artery-cameral fistula ( $0.1 \%)$. Minor feeders came from the conal branch of the RCA while the major feeder was from the septal branches of the LAD. Both feeders were connected to the common vascular channel that was finally draining into to the PA. The conal branch of RCA had rather simple straight away course upwards towards the common channel while the septal branch had tortuous irregular course along the left lateral aspect of the main PA. The diameter of the LAD was relatively increased as it is the primary feeder of the fistula. Likewise, tortuosity was reported in another study [29] to occur with older age, which copes well with our 52-year-old case. Clinical symptoms are proportional to the amount of induced haemodynamic abnormality. Patients with small CA fistulas remain asymptomatic, whereas those with high-flow fistulas may develop haemodynamic steal phenomenon with consequent myocardial ischaemia [43].

Coronary artery "dominance" is defined in terms of which CA supplies the posterior descending artery (PDA, also known as the "inferior interventricular artery"), and the posterior left ventricular branches. The PDA is the artery that extends along the inferior interventricular groove, from the crux of the heart toward the apex. If the PDA is supplied by the RCA, then the coronary circulation can be classified as "right-dominant". If the PDA is supplied by the circumflex artery (CX), a branch of the left artery, then the coronary circulation can be classified as "left-dominant" [17]. However, if the PDA is supplied by both the RCA and the CX, then the coronary circulation can be classified as "co-dominant". Approximately $70 \%$ of the general population are right-dominant, $20 \%$ are co-dominant, and $10 \%$ are left-dominant. A precise anatomic definition of dominance would be the artery that gives off supply to the $A V$ node i.e. the $A V$ nodal artery. Most of the time this is the RCA [17]. In our study, $83.8 \%$ of cases showed RCA dominance while $9 \%$ and $7.2 \%$ showed left and co-dominance, respectively. These data are in agreement with previously published studies $[8,25]$.

The ramus intermedius (Fig. 8) is a variant CA resulting from trifurcation of the main left CA. It is present in about $20 \%$ (range 15-30\%) of the population $[13,28]$. Moreover, it is considered the most common anatomic variation observed in the left coronary system [13]. In the current study, it was found in $19 \%$ of the cases.

This study has several limitations that artificially may have raised the incidence of the anomalies reported. One limitation was the retrospective design of the trial, which was prone to confounding factors and bias. Moreover, the small sample size and the study population was highly selected our sample does not represent the true prevalence in a more general population since the cases were collected from a single hospital in Kuwait. Further, the study may have included patients included that underwent CT because of an anomaly that was seen or suspected in cath (a not-so-infrequent reason for referral). Surgical confirmation of CTA finding was not available since none of them underwent a surgical correction, especially those who are with anomalous origin and fistula.

\section{CONCLUSIONS}

The prevalence of CAA in the subjects referred to our institution in Kuwait was $2.6 \%$ that is relatively higher than the average percentage reported in the literature. A further elaborate follow-up study is needed to analyse the exact prevalence of congenital coronary anomalies in the whole Kuwaiti pupation. 


\section{REFERENCES}

1. Achenbach S, Marwan M, Ropers D, et al. Coronary computed tomography angiography with a consistent dose below $1 \mathrm{mSv}$ using prospectively electrocardiogramtriggered high-pitch spiral acquisition. Eur Heart J. 2010; 31(3): 340-346, doi: 10.1093/eurheartj/ehp470, indexed in Pubmed: 19897497.

2. Aldana-Sepulveda N, Restrepo CS, Kimura-Hayama E. Single coronary artery: spectrum of imaging findings with multidetector CT. J Cardiovasc Comput Tomogr. 2013; 7(6): 391-399, doi: 10.1016/j.jcct.2013.11.009, indexed in Pubmed: 24331935.

3. Alegria JR, Herrmann J, Holmes DR, et al. Myocardial bridging. Eur Heart J. 2005; 26(12): 1159-1168, doi: 10.1093/ eurheartj/ehi203, indexed in Pubmed: 15764618.

4. Angelini P, Velasco JA, Flamm S. Coronary anomalies: incidence, pathophysiology, and clinical relevance. Circulation. 2002; 105(20): 2449-2454, indexed in Pubmed: 12021235.

5. Angelini P. Coronary artery anomalies: an entity in search of an identity. Circulation. 2007; 115(10): 1296-1305, doi: 10.1161/CIRCULATIONAHA.106.618082, indexed in Pubmed: 17353457.

6. Armsby LR, Keane JF, Sherwood MC, et al. Management of coronary artery fistulae. Patient selection and results of transcatheter closure. J Am Coll Cardiol. 2002; 39(6): 1026-1032, indexed in Pubmed: 11897446.

7. Basso C, Maron BJ, Corrado D, et al. Clinical profile of congenital coronary artery anomalies with origin from the wrong aortic sinus leading to sudden death in young competitive athletes. J Am Coll Cardiol. 2000; 35(6): 1493-1501, indexed in Pubmed: 10807452.

8. Cademartiri F, La Grutta L, Malagò R, et al. Prevalence of anatomical variants and coronary anomalies in 543 consecutive patients studied with 64-slice CT coronary angiography. Eur Radiol. 2008; 18(4): 781-791, doi: 10.1007/ s00330-007-0821-9, indexed in Pubmed: 18246357.

9. Cho SH, Joo HC, Yoo KJ, et al. Anomalous origin of right coronary artery from left coronary sinus: surgical management and clinical result. Thorac Cardiovasc Surg. 2015; 63(5): 360-366, doi: 10.1055/s-0034-1376256, indexed in Pubmed: 24911900.

10. Click RL, Holmes DR, Vlietstra RE, et al. Anomalous coronary arteries: location, degree of atherosclerosis and effect on survival: a report from the Coronary Artery Surgery Study. J Am Coll Cardiol. 1989; 13(3): 531-537, indexed in Pubmed: 2918156.

11. Danias PG, Stuber M, McConnell MV, et al. The diagnosis of congenital coronary anomalies with magnetic resonance imaging. Coron Artery Dis. 2001; 12(8): 621-626, indexed in Pubmed: 11811327.

12. Desmet $\mathrm{W}$, Vanhaecke J, Vrolix $\mathrm{M}$, et al. Isolated single coronary artery: a review of 50,000 consecutive coronary angiographies. Eur Heart J. 1992; 13(12): 1637-1640, indexed in Pubmed: 1289093.

13. Dewey M, Kroft UM. Anatomy. In: Dewey M. ed. Coronary CT angiography. Springer, Berlin 2009: 11-26.

14. Dhoble A, Dewar J, Szerlip M, et al. Rare coronary anomaly: common origin of major coronary arteries from the right sinus of Valsalva and a small ramus branch originating from the left sinus of Valsalva. Int J Cardiol. 2012; 158(1): e3-e4, doi: 10.1016/j.ijcard.2011.10.062, indexed in Pubmed: 22104988.

15. Dodd JD, Ferencik M, Liberthson RR, et al. Congenital anomalies of coronary artery origin in adults: $64-\mathrm{MDCT}$ appearance. AJR Am J Roentgenol. 2007; 188(2): W138-W146, doi: 10.2214/AJR.05.2098, indexed in Pubmed: 17242219.

16. Dodge-Khatami A, Mavroudis C, Backer CL. Congenital heart surgery nomenclature and database project: Anomalies of the coronary arteries. Ann Thorac Surg. 2000; 69(4 Suppl): S270-S297, indexed in Pubmed: 10798435.

17. Fuster V, Alexander RW, O'Rourke RA. Hurst's The Heart (10th ed.). McGraw-Hill, New York 2001: 53.

18. Ghadri JR, Küest SM, Goetti R, et al. Image quality and radiation dose comparison of prospectively triggered lowdose CCTA: 128-slice dual-source high-pitch spiral versus 64-slice single-source sequential acquisition. Int J Cardiovasc Imaging. 2012; 28(5): 1217-1225, doi: 10.1007/ s10554-011-9921-3, indexed in Pubmed: 21744246.

19. Herzog BA, Wyss CA, Husmann L, et al. First head-tohead comparison of effective radiation dose from lowdose 64-slice CT with prospective ECG-triggering versus invasive coronary angiography. Heart. 2009; 95(20): 1656-1661, doi: 10.1136/hrt.2008.162420, indexed in Pubmed: 19581273.

20. Ishikawa T, Brandt PW. Anomalous origin of the left main coronary artery from the right anterior aortic sinus: angiographic definition of anomalous course. Am J Cardiol. 1985; 55(6): 770-776, indexed in Pubmed: 3976523.

21. Ishikawa $Y$, Kawawa $Y$, Kohda $E$, et al. Significance of the anatomical properties of a myocardial bridge in coronary heart disease. Circ J. 2011; 75(7): 1559-1566, indexed in Pubmed: 21467656.

22. Kacmaz F, Ozbulbul NI, Alyan O, et al. Imaging of coronary artery anomalies: the role of multidetector computed tomography. Coron Artery Dis. 2008; 19(3): 203-209, doi: 10.1097/MCA.0b013e3282f528f1, indexed in Pubmed: 18418238.

23. Kim PJ, Hur G, Kim SuY, et al. Frequency of myocardial bridges and dynamic compression of epicardial coronary arteries: a comparison between computed tomography and invasive coronary angiography. Circulation. 2009; 119(10): 1408-1416, doi: 10.1161/CIRCULATIONAHA.108.788901, indexed in Pubmed: 19255347.

24. Kim SY, Seo JB, Do KH, et al. Coronary artery anomalies: Classification and ECG-gated multi-detector row CT findings with angiographic correlation. Radiographics. 2006; 26(2): 317-333, doi: 10.1148/rg.262055068, indexed in Pubmed: 16549600.

25. Kini S, Bis KG, Weaver L. Normal and variant coronary arterial and venous anatomy on high-resolution CT angiography. AJR Am J Roentgenol. 2007; 188(6): 1665-1674, doi: 10.2214/AJR.06.1295, indexed in Pubmed: 17515392.

26. Konen E, Goitein O, Sternik L, et al. The prevalence and anatomical patterns of intramuscular coronary arteries: A coronary computed tomography angiographic study. J Am Coll Cardiol. 2007; 49(5): 587-593, doi: 10.1016/j. jacc.2006.09.039, indexed in Pubmed: 17276183.

27. Koşar $P$, Ergun $E$, Oztürk $C$, et al. Anatomic variations and anomalies of the coronary arteries: 64-slice CT angiographic appearance. Diagn Interv Radiol. 2009; 15(4): 
275-283, doi: 10.4261/1305-3825.DIR.2550-09.1, indexed in Pubmed: 19957241.

28. Krupiński M, Urbańczyk-Zawadzka M, Laskowicz B, et al. Anomalous origin of the coronary artery from the wrong coronary sinus evaluated with computed tomography: "high-risk" anatomy and its clinical relevance. Eur Radiol. 2014; 24(10): 2353-2359, doi: 10.1007/s00330-014-3238-2, indexed in Pubmed: 24895033.

29. Manghat NE, Morgan-Hughes GJ, Marshall AJ, et al. Multidetector row computed tomography: imaging congenital coronary artery anomalies in adults. Heart. 2005; 91(12): 1515-1522, doi: 10.1136/hrt.2005.065979, indexed in Pubmed: 16287728 .

30. Nakanishi R, Rajani R, Ishikawa $Y$, et al. Myocardial bridging on coronary CTA: An innocent bystander or a culprit in myocardial infarction? J Cardiovasc Comput Tomogr. 2012; 6(1): 3-13, doi: 10.1016/j.jcct.2011.10.015, indexed in Pubmed: 22264630.

31. Namgung J, Kim JA. The prevalence of coronary anomalies in a single center of Korea: origination, course, and termination anomalies of aberrant coronary arteries detected by ECG-gated cardiac MDCT. BMC Cardiovasc Disord. 2014; 14: 48, doi: 10.1186/1471-2261-14-48, indexed in Pubmed: 24725604.

32. Patel KB, Gupta $H$, Nath $H$, et al. Origin of all three major coronary arteries from the right sinus of Valsalva: clinical, angiographic, and magnetic resonance imaging findings and incidence in a select referral population. Catheter Cardiovasc Interv. 2007; 69(5): 711-718, doi: 10.1002/ ccd.21078, indexed in Pubmed: 17330267.

33. Schroeder S, Achenbach S, Bengel F, et al. Working Group Nuclear Cardiology and Cardiac CT, European Society of Cardiology, European Council of Nuclear Cardiology. Cardiac computed tomography: indications, applications, limitations, and training requirements: report of a Writing Group deployed by the Working Group Nuclear Cardiology and Cardiac CT of the European Society of Cardiology and the European Council of Nuclear Cardiology. Eur Heart J. 2008; 29(4): 531-556, doi: 10.1093/eurheartj/ehm544, indexed in Pubmed: 18084017.

34. Shabestari AA, Akhlaghpoor S, Tayebivaljozi R, et al. Prevalence of congenital coronary artery anomalies and variants in 2697 consecutive patients using 64-detector row coronary ctangiography. Iran J Radiol. 2012; 9(3): 111-121, doi: 10.5812/iranjradiol.8070, indexed in Pubmed: 23329976
35. Shi H, Aschoff AJ, Brambs HJ, et al. Multislice CT imaging of anomalous coronary arteries. Eur Radiol. 2004; 14(12): 2172-2181, doi: 10.1007/s00330-004-2490-2, indexed in Pubmed: 15490179.

36. Sundaram B, Kreml R, Patel S. Imaging of coronary artery anomalies. Radiol Clin North Am. 2010; 48(4): 711-727, doi: 10.1016/j.rcl.2010.04.006, indexed in Pubmed: 20705168.

37. Taylor AJ, Rogan KM, Virmani R. Sudden cardiac death associated with isolated congenital coronary artery anomalies. J Am Coll Cardiol. 1992; 20(3): 640-647, indexed in Pubmed: 1512344.

38. von Ziegler F, Pilla M, McMullan L, et al. Visualization of anomalous origin and course of coronary arteries in 748 consecutive symptomatic patients by 64 -slice computed tomography angiography. BMC Cardiovasc Disord. 2009; 9: 54, doi: 10.1186/1471-2261-9-54, indexed in Pubmed: 20003347.

39. Won YJ, Kim HJ, Lee H. Unexpected sudden death of a 19-year-old female with congenital single coronary artery ostium during exertion. Yonsei Med J. 2011; 52(5): 856-858, doi: 10.3349/ymj.2011.52.5.856, indexed in Pubmed: 21786453.

40. Xu H, Zhu Y, Zhu X, et al. Anomalous coronary arteries: depiction at dual-source computed tomographic coronary angiography. J Thorac Cardiovasc Surg. 2012; 143(6): 1286-1291, doi: 10.1016/j.jtcvs.2011.11.025, indexed in Pubmed: 22154794.

41. Young PM, Gerber TC, Williamson EE, et al. Cardiac imaging: Part 2, normal, variant, and anomalous configurations of the coronary vasculature. AJR Am J Roentgenol. 2011; 197(4): 816-826, doi: 10.2214/AJR.10.7249, indexed in Pubmed: 21940568.

42. Zeina AR, Blinder J, Sharif $D$, et al. Congenital coronary artery anomalies in adults: non-invasive assessment with multidetector CT. Br J Radiol. 2009; 82(975): 254-261, doi: 10.1259/bjr/80369775, indexed in Pubmed: 19001466.

43. Zenooz NA, Habibi R, Mammen L, et al. Coronary artery fistulas: CT findings. Radiographics. 2009; 29(3): 781-789, doi: 10.1148/rg.293085120, indexed in Pubmed: 19448115.

44. Zhou CS, Zhang $\sqcup$, Wang Y, et al. [Incidence and classification diagnosis of coronary artery fistula at dual-source $C T$ coronary angiography]. Zhonghua Yi Xue Za Zhi. 2012; 92(47): 3336-3340, indexed in Pubmed: 23328594. 